\title{
ON THE MAXIMUM OF SUMS OF RANDOM VARIABLES AND THE SUPREMUM FUNCTIONAL FOR STABLE PROCESSES
}

\author{
C. C. HEYDE*, University of Manchester
}

\section{Introduction}

Let $X_{i}, i=1,2,3, \cdots$ be a sequence of independent and identically distributed random variables which belong to the domain of attraction of a stable law of index $\alpha$. Write $S_{0}=0, S_{n}=\sum_{i=1}^{n} X_{i}, n \geqq 1$, and $M_{n}=\max _{0 \leqq k \leqq n} S_{k}$. In the case where the $X_{i}$ are such that $\sum_{1}^{\infty} n^{-1} \operatorname{Pr}\left(S_{n}>0\right)<\infty$, we have $\lim _{n \rightarrow \infty} M_{n}=M$ which is finite with probability one, while in the case where $\sum_{1}^{\infty} n^{-1} \operatorname{Pr}\left(S_{n}<0\right)<\infty$, a limit theorem for $M_{n}$ has been obtained by Heyde [9]. The techniques used in [9], however, break down in the case $\sum_{1}^{\infty} n^{-1} \operatorname{Pr}\left(S_{n}<0\right)=\infty, \sum_{1}^{\infty} n^{-1} \operatorname{Pr}\left(S_{n}>0\right)=\infty$ (the case of oscillation of the random walk generated by the $S_{n}$ ) and the only results available deal with the case $\alpha=2$ (Erdös and Kac [5]) and the case where the $X_{i}$ themselves have a symmetric stable distribution (Darling [4]). In this paper we obtain a general limit theorem for $M_{n}$ in the case of oscillation. Specifically, if $\left\{B_{n}, n=1,2,3, \cdots\right\}$ is a monotone sequence of constants such that $B_{n}^{-1} S_{n}$ converges in distribution to the stable law with characteristic function

$$
\exp \left\{-\lambda|t|^{\alpha}\left(1+i \beta \operatorname{sgn} t \tan \frac{\pi \alpha}{2}\right)\right\}
$$

$\lambda>0,0<\alpha \leqq 2, \beta=0$ if $\alpha=1,|\beta|<1$ if $\alpha<1,|\beta| \leqq 1$ if $1<\alpha \leqq 2$, we shall find

$$
H(x)=\lim _{n \rightarrow \infty} \operatorname{Pr}\left(B_{n}^{-1} M_{n} \leqq x\right) .
$$

In connection with the parameter restrictions, we note that the stable law with characteristic function (1) is one-sided if $\alpha<1,|\beta|=1$ (e.g., Lukacs [12], page 106) so that the random walk generated by the $S_{n}$ does not oscillate ([9], Lemma). The case $\alpha=1, \beta \neq 0$ introduces a normalization complication and is not amenable to treatment by the methods of this paper.

It is possible to approach the problem of finding $H(x)$ in various ways. For, if $Y(t), t \geqq 0, Y(0)=0$, is the separable stable process with stationary independent increments which is based on (1), then also,

Received in revised form 26 August 1968.

* Now at the Australian National University. 


$$
H(x)=\operatorname{Pr}\left(\sup _{0 \leqq t \leqq 1} Y(t)<x\right) .
$$

The double Laplace transform of $\operatorname{Pr}\left(\sup _{0 \leqq u \leqq t} Y(u)<x\right)$ has, in this context, been obtained by Baxter and Donsker [1]. Their results are, however, not in a sufficiently explicit form to enable any of the properties of $H(x)$ to be deduced. In the present work, we make a more detailed investigation leading to considerably more illuminating results.

\section{The supremum functional for a stable process}

In this section, we shall deal with the case where the $X_{i}$ themselves have a stable distribution with characteristic function (1) (and, of course, the specified parameter restrictions). This will be followed in the next section by an invariance theorem to establish the generality of the limiting distribution found in the present context.

Under the present circumstances, it is a simple matter to establish the existence of a limiting distribution for $n^{-1 / \alpha} M_{n}$. In fact, if $Y(t), t \geqq 0, Y(0)=0$, is the separable stable process with stationary independent increments which is characterised by

$$
E \exp \{i u Y(T)\}=\exp \left\{-T \lambda|u|^{\alpha}\left(1+i \beta \operatorname{sgn} u \tan \frac{\pi \alpha}{2}\right)\right\},
$$

and if we take

$$
X_{k}=\left[Y\left(\frac{k}{2^{N}}\right)-Y\left(\frac{k-1}{2^{N}}\right)\right] 2^{N \alpha^{-1}}, \quad k=1,2, \cdots, 2^{N},
$$

then it follows readily from Lemma 2 of Baxter and Donsker [1], that

$$
\lim _{n \rightarrow \infty} \operatorname{Pr}\left(n^{-1 / \alpha} M_{n}<x\right)=H(x),
$$

where

$$
H(x)=\operatorname{Pr}\left(\sup _{0 \leqq t \leqq 1} Y(t)<x\right)
$$

and also

$$
\operatorname{Pr}\left(\sup _{0 \leqq t \leqq T} Y(t)<x\right)=H\left(x T^{-1 / \alpha}\right) .
$$

We shall proceed, using methods of Darling [4], to obtain an expression for $H(x)$.

For $s$ real and positive, let

$$
\begin{aligned}
\phi_{n}(s) & =\operatorname{Pr}\left(S_{n} \leqq 0\right)+E\left(\exp \{-s S n\} ; S_{n}>0\right) \\
& =\operatorname{Pr}(X \leqq 0)+E\left(\exp \left\{-s n^{1 / \alpha} X\right\} ; X>0\right),
\end{aligned}
$$

since $n^{-1 / \alpha} S_{n}$ and $X_{i}$ have the same distribution. Then, using Theorem 4 of Zolotarev [18], we obtain the unilateral Laplace transform 


$$
E\left(e^{-s X} ; X>0\right)=\frac{1}{\pi} \int_{0}^{\infty} \exp \left\{-(s x)^{\alpha} \lambda_{1}\right\} \frac{\sin \pi \rho}{x^{2}+2 x \cos \pi \rho+1} d x,
$$

where

(5) $\lambda_{1}=\lambda\left(1+\beta^{2} \tan ^{2} \frac{\pi \alpha}{2}\right)^{1 / 2}, \rho=\frac{1}{2}+\frac{1}{\pi \alpha} \arctan \left[-\beta \tan \frac{\pi \alpha}{2}\right]=\operatorname{Pr}(X>0)$, (the result $\rho=\operatorname{Pr}(X>0)$ was obtained by Chung-Teh [2]), so that for $0 \leqq t<1$,

$$
\begin{aligned}
& \sum_{1}^{\infty} n^{-1} t^{n} \phi_{n}(s) \\
= & -(1-\rho) \log (1-t)-\frac{\sin \pi \rho}{\pi} \int_{0}^{\infty} \log \left[1-t \exp \left\{-(s x)^{\alpha} \lambda_{1}\right\} \frac{d x}{x^{2}+2 x \cos \pi \rho+1} .\right.
\end{aligned}
$$

But, using (4),

$$
\frac{\sin \pi \rho}{\pi} \int_{0}^{\infty} \frac{d x}{x^{2}+2 x \cos \pi \rho+1}=\rho
$$

and therefore,

$$
\begin{aligned}
\log (1-t) & +\sum_{1}^{\infty} n^{-1} t^{n} \phi_{n}(s) \\
& =-\frac{\sin \pi \rho}{\pi} \int_{0}^{\infty} \log \left[\frac{1-t \exp \left\{-(s x)^{\alpha} \lambda_{1}\right\}}{1-t}\right] \frac{d x}{x^{2}+2 x \cos \pi \rho+1} .
\end{aligned}
$$

Consequently, as $t \uparrow 1$,

$$
\log (1-t)+\sum_{1}^{\infty} n^{-1} t^{n} \phi_{n}\left[(1-t)^{1 / \alpha} s\right] \rightarrow-\frac{\sin \pi \rho}{\pi} \int_{0}^{\infty} \frac{\log \left(1+s^{\alpha} x^{\alpha} \lambda_{1}\right)}{x^{2}+2 x \cos \pi \rho+1} d x
$$

and, using the Spitzer-Pollaczek Identity (see for example Prabhu [13], page 218, Theorem 4.1),

$$
\lim _{t \uparrow 1}(1-t) \sum_{0}^{\infty} E\left(\exp \left\{-s(1-t)^{1 / \alpha}\right\} M_{n}\right)=g(s)
$$

where

$$
g(s)=\exp \left\{-\frac{\sin \pi \rho}{\pi} \int_{0}^{\infty} \frac{\log \left(1+s^{\alpha} x^{\alpha} \lambda_{1}\right)}{x^{2}+2 x \cos \pi \rho+1} d x\right\} .
$$

Precisely the argument of Darling [4] then yields the relation 


$$
A(s)=\frac{G(1-s)}{\Gamma(1-s) \Gamma\left(1-\alpha^{-1}+s \alpha^{-1}\right)},
$$

$|\mathscr{R} s|$ sufficiently small, between the Mellin transforms,

$$
\begin{aligned}
& A(s)=\int_{0}^{\infty} x^{s-1} d H(x), \\
& G(s)=\int_{0}^{\infty} x^{s-1} g(x) d x .
\end{aligned}
$$

As with [4], it follows that $H(x)$ is an absolutely continuous distribution function and upon inverting in (8) we obtain the density function

$$
\frac{d}{d x} H(x)=\frac{1}{2 \pi i} \int_{c-i \infty}^{c+i \infty} \frac{G(1-s) x^{-s}}{\Gamma(1-s) \Gamma\left(1-\alpha^{-1}+s \alpha^{-1}\right)} d s,
$$

(see for example Widder [17], pages 246, 247). We have thus established the following theorem.

Theorem 1. If $0<\alpha \leqq 2$ and $\beta=0$ if $\alpha=1,|\beta|<1$ if $\alpha<1,|\beta| \leqq 1$ if $1<\alpha \leqq 2$, then

$$
\frac{d}{d x} H(x)=\frac{1}{2 \pi i} \int_{c-i \infty}^{c+i \infty} \frac{G(1-s) x^{-s}}{\Gamma(1-s) \Gamma\left(1-\alpha^{-1}+s \alpha^{-1}\right)} d s
$$

where

$$
G(s)=\int_{0}^{\infty} x^{s-1} g(x) d x
$$

$g(x)$ being given by (7).

The expression in (9) does not in general simplify conveniently. We shall go on to examine an alternative method which is illuminating in its own right and has the advantage of providing more recognisable results in certain particular cases. This is based on the representation of $M_{n}$ in the form

$$
\begin{aligned}
& M_{n}=0 \text { if } R_{n}=0 \\
& M_{n}=Z_{1}+Z_{2}+\cdots+Z_{R_{n}}, \text { if } R_{n} \geqq 1,
\end{aligned}
$$

where the $Z_{i}$ are successive strong ascending ladder steps for the random walk generated by the $S_{n}$ (that is, $Z_{i}$ has the distribution of the minimum $S_{k}$ with $S_{k}>0$ ) and $R_{n}$ is the number of strong ascending ladder indices in the first $n$ steps of the random walk. 
Firstly, we need to investigate the distribution of $R_{n}$. We easily see that the occurrence of a strong ascending ladder index is a recurrent event in the usual sense of Feller. This we shall denote by $\mathscr{E}$, so that $R_{n}$ is the number of occurrences of $\mathscr{E}$ in $n$ steps. $R_{n}$ can be expressed as a sum of indicator random variables in the following way:

where

$$
R_{n}=\sum_{k=1}^{n} \delta_{k}
$$

$$
\delta_{k}=\left\{\begin{array}{l}
1 \text { if } \mathscr{E} \text { occurs at the } k \text { th step, } \\
0 \text { otherwise }
\end{array}\right.
$$

and we can study $R_{n}$ via the $\delta_{k}$. If $N_{n}$ is the number of positive terms in the sequence $S_{1}, S_{2}, \cdots, S_{n}$,

$$
\begin{aligned}
\operatorname{Pr}\left(\delta_{k}=1\right) & =\operatorname{Pr}\left(S_{k}>0, \quad S_{k}>S_{1}, \cdots, S_{k}>S_{k-1}\right) \\
& =\operatorname{Pr}\left(S_{k}>0, \quad \sum_{2}^{k} X_{i}>0, \cdots, X_{k}>0\right) \\
& =\operatorname{Pr}\left(N_{k}=k\right),
\end{aligned}
$$

the $X_{i}$ being identically distributed. We now define sequences $\left\{u_{n}, n \geqq 0\right\}$, $\left\{f_{n}, n \geqq 1\right\}$, as follows:

$$
\begin{aligned}
& u_{0}=1, \quad u_{n}=\operatorname{Pr}\left(\delta_{n}=1\right), \quad n \geqq 1, \\
& f_{n}=\operatorname{Pr}\left(\delta_{1}=0, \delta_{2}=0, \cdots, \delta_{n-1}=0, \delta_{n}=1\right), \quad n \geqq 1,
\end{aligned}
$$

and introduce the generating functions

$$
U(t)=\sum_{n=0}^{\infty} u_{n} t^{n}, \quad F(t)=\sum_{n=1}^{\infty} f_{n} t^{n}, \quad 0 \leqq t<1 .
$$

These, of course, satisfy the standard identity of recurrent event theory,

$$
U(t)=[1-F(t)]^{-1}, \quad 0 \leqq t<1 .
$$

Then, using a well-known result of Sparre-Andersen (see for example Spitzer [16], page 219) together with (5) and (10), we have

$$
U(t)=\exp \left\{\sum_{1}^{\infty} t^{k} k^{-1} \operatorname{Pr}(X>0)\right\}=(1-t)^{-\rho} .
$$

Lemma. The recurrence time distribution of $\mathscr{E}$ belongs to the domain of attraction of the stable law of index $1-\rho$. 
Proof. If $q_{n}=\sum_{r=n+1}^{\infty} f_{r}$, we have

$$
(1-t)^{-1}[1-F(t)]=\sum_{n=0}^{\infty} q_{n} t^{n}=(1-t)^{-(1-\rho)},
$$

using (11) and (12), so that

$$
(1-t)^{1-\rho} \sum_{n=0}^{\infty} q_{n} t^{n}=1
$$

and applying a standard Tauberian theorem (see for example Feller [7], page 423, Theorem 5),

$$
q_{n} \sim \frac{1}{\Gamma(1-\rho)} n^{-\rho}
$$

as $n \rightarrow \infty$. The result of the lemma then follows immediately by appeal to Theorem la, page 303 of Feller [7]. Then, using the lemma in conjunction with Theorem 7 of Feller [6], we readily obtain:

Theorem 2. Let $G_{\rho}$ be the distribution function of the stable law whose characteristic function is given by

$$
g_{\rho}(t)=\exp \left\{-|t|^{\rho}\left(\cos \frac{\pi \rho}{2}-i \sin \frac{\pi \rho}{2} \operatorname{sgn} t\right)\right\},
$$

where $\rho=\operatorname{Pr}(X>0)$. Then,

$$
\lim _{n \rightarrow \infty} \operatorname{Pr}\left(n^{-\rho} R_{n} \leqq x\right)=1-G_{\rho}\left(x^{-1 / \rho}\right), \quad x \geqq 0 .
$$

Remark. Only in the case $\rho=\frac{1}{2}$ is it possible to give a convenient alternative form for the limit distribution in (13). We have, making use of a result due to Lévy (see for example Lukacs [12], page 107),

$$
\frac{d}{d x} G_{\frac{1}{2}}(x)=\frac{1}{2} \pi^{-\frac{1}{2}} x^{-\frac{3}{2}} \exp \{-1 / 4 x\}, \quad x>0
$$

so that

$$
\frac{d}{d x}\left[1-G_{\frac{1}{2}}\left(x^{-2}\right)\right]=\pi^{-\frac{1}{2}} \exp \left\{-1 / 4 x^{2}\right\}
$$

Thus, the limit distribution is a truncated normal.

Next, we need to examine the limit properties of the $Z_{i}$. We have (see for example Prabhu [13], page 210, Theorem 2.2) 


$$
\begin{aligned}
1-E e^{-s Z} & =\exp \left\{-\sum_{1}^{\infty} n^{-1} E\left(\exp \{-s S n\} ; S_{n}>0\right)\right\} \\
& =\exp \left\{-\sum_{1}^{\infty} n^{-1} E\left(\exp \left\{-s n^{1 / \alpha} X\right\} ; X>0\right)\right\} \\
& =\exp \left\{\frac{\sin \pi \rho}{\pi} \int_{0}^{\infty} \log \left[1-\exp \left\{-(s x)^{\alpha} \lambda_{1}\right\}\right] \frac{d x}{x^{2}+2 x \cos \pi \rho+1}\right\}
\end{aligned}
$$

using (4). Furthermore, if

$$
I(s)=\int_{0}^{\infty} \log \left[1-\exp \left\{-(s x)^{\alpha} \lambda_{1}\right\}\right] \frac{d x}{x^{2}+2 x \cos \pi \rho+1},
$$

then we can write $I(s)=I_{1}(s)+I_{2}(s)$, where

$$
I_{1}(s)=\int_{0}^{\infty} \frac{\log b(s x)}{x^{2}+2 x \cos \pi \rho+1} d x, \quad I_{2}(s)=\int_{0}^{\infty} \frac{\log \left[(s x)^{\alpha} \lambda_{1}\right]}{x^{2}+2 x \cos \pi \rho+1} d x,
$$

and $b(u)=\left[1-\exp \left\{-u^{\alpha} \lambda_{1}\right\}\right] /\left(u^{\alpha} \lambda_{1}\right)$. Then, $I_{1}(s) \rightarrow 0$ as $s \rightarrow 0$ using dominated convergence. Also,

$$
\int_{0}^{\infty} \frac{d x}{x^{2}+2 x \cos \pi \rho+1}=\frac{\pi \rho}{\sin \pi \rho}, \quad \int_{0}^{\infty} \frac{\log x d x}{x^{2}+2 x \cos \pi \rho+1}=0
$$

using (4) and Gradshteyn and Ryzhik [8], page 533, respectively, so that

and hence

$$
I_{2}(s)=\frac{\pi \rho}{\sin \pi \rho}\left(\log \lambda_{1}+\alpha \log s\right)
$$

$$
\begin{aligned}
s^{-\alpha \rho}\left(1-E e^{-s Z}\right) & =s^{-\alpha \rho} \exp \left\{\frac{\sin \pi \rho}{\pi}\left[I_{1}(s)+I_{2}(s)\right]\right\} \\
& =\lambda_{1}^{\rho} \exp \left\{\frac{\sin \pi \rho}{\pi} I_{1}(s)\right\} \\
& \rightarrow \lambda_{1}^{\rho}=\left[\lambda\left(1+\beta^{2} \tan ^{2} \frac{\pi \alpha}{2}\right)^{\frac{1}{2}}\right]^{\operatorname{Pr}(X>0)}
\end{aligned}
$$

as $s \rightarrow 0$. Now it is easily checked from (5) that $\alpha \rho \leqq 1$, with equality if and only if $1<\alpha \leqq 2$ and $\beta=1$ when $\alpha<2$. Thus, if $0<\alpha \leqq 1$ or $1<\alpha<2$ and $-1 \leqq \beta<1$, $n^{-1 / \alpha \rho}\left(Z_{1}+\cdots+Z_{n}\right)$ converges in distribution to the stable law with characteristic function

$$
\exp \left\{-\lambda_{1}^{\rho}|t|^{\alpha \rho}\left(1-i \operatorname{sgn} t \tan \frac{\pi \alpha \rho}{2}\right)\right\},
$$

while if $1<\alpha \leqq 2$ and $\beta=1$ when $\alpha<2$, we have $\alpha \rho=1$ so that 


$$
s^{-1}\left(1-E e^{-s z}\right) \rightarrow\left[\lambda\left(1+\tan ^{2} \frac{\pi \alpha}{2}\right)^{\frac{1}{2}}\right]^{1 / \alpha}=\left[\lambda \sec \left(\pi-\frac{\pi \alpha}{2}\right)\right]^{1 / \alpha},
$$

and therefore,

$$
E Z=\left[\lambda \sec \left(\pi-\frac{\pi \alpha}{2}\right)\right]^{1 / \alpha}<\infty
$$

These results clarify those of Rogozin [15], where it is not possible to calculate specifically the constant in (15). We are now in a position to establish the following limiting result.

Theorem 3. If $1<\alpha \leqq 2$ and $\beta=1$ when $\alpha<2,|\beta| \leqq 1$ when $\alpha=2$, then

$$
H(x)=1-G_{1 / \alpha}\left(-\lambda \sec \frac{\pi \alpha}{2} x^{-\alpha}\right), \quad x \geqq 0,
$$

where $G_{1 / \alpha}$ is the distribution function of the stable law whose characteristic function is given by

$$
g_{1 / \alpha}(t)=\exp \left\{-|t|^{1 / \alpha}\left(\cos \frac{\pi}{2 \alpha}-i \sin \frac{\pi}{2 \alpha} \operatorname{sgn} t\right)\right\} .
$$

Proof. We may write

$$
\frac{M_{n}}{n^{1 / \alpha}}=\frac{Z_{1}+\cdots+Z_{R_{n}}}{n^{1 / \alpha}}=\frac{R_{n}}{n^{1 / \alpha}} \cdot \frac{Z_{1}+\cdots+Z_{R_{n}}}{R_{n}} .
$$

Now, in view of (16) and using Theorem 2 of Richter [14],

$$
\frac{Z_{1}+\cdots+Z_{R_{n}}}{R_{n}} \stackrel{p}{\rightarrow} E Z=\left[\lambda \sec \left(\pi-\frac{\pi \alpha}{2}\right)\right]^{1 / \alpha},
$$

(" $p$ " stands for convergence in probability). Also, we have from Theorem 1 that

$$
\lim _{n \rightarrow \infty} \operatorname{Pr}\left(n^{-1 / \alpha} R_{n} \leqq x\right)=1-G_{1 / \alpha}\left(x^{-\alpha}\right) .
$$

Then, using a standard convergence result (see for example Cramér [3], page 254), it follows from (18), (19) and (20) that

$$
\begin{aligned}
H(x)=\lim _{n \rightarrow \infty} \operatorname{Pr}\left(n^{-1 / \alpha} M_{n} \leqq x\right) & =\lim _{n \rightarrow \infty} \operatorname{Pr}\left(n^{-1 / \alpha} R_{n} E Z \leqq x\right) \\
& =1-G_{1 / \alpha}\left(-\lambda \sec \frac{\pi \alpha}{2} x^{-\alpha}\right) .
\end{aligned}
$$

This completes the proof of the theorem. In the particular case $\alpha=2$, we see from (14) that

$$
H(x)=\frac{1}{\sqrt{ } \pi} \int_{0}^{x / \sqrt{ } \lambda} e^{-\frac{1}{4} u 2} d u=\frac{1}{\sqrt{ }(\pi \lambda)} \int_{0}^{x} e^{-u^{2} / 4 \lambda} d u .
$$


The result (21) is well known and has a long history. Different methods of derivation can be seen in Erdös and Kac [5], Lévy [11], page 85, and Darling [4].

\section{Limit theorem for maxima of partial sums}

We shall establish the following invariance theorem.

Theorem 4. Let $X_{i}, i=1,2,3, \cdots$ be a sequence of independent and identically distributed random variables and write $S_{n}=\sum_{i=1}{ }_{n} X_{i}, n \geqq 1, \quad S_{0}=0$, and $M_{n}=\max _{0 \leqq k \leqq n} S_{k}$. Suppose that there exists a monotone sequence of constants $\left\{B_{n}, n=1,2,3, \cdots\right\}$ such that $B_{n}^{-1} S_{n}$ converges in distribution to the stable law with characteristic function (1). Then,

$$
\lim _{n \rightarrow \infty} \operatorname{Pr}\left(M_{n} \leqq B_{n} x\right)=H(x),
$$

the distribution function $H(x)$ being given by (9), (17) or (21).

Proof. Let $Y_{i}, i=1,2,3, \cdots$ be independent and identically distributed random variables with characteristic function (1) and write $W_{n}=\sum_{i=1}^{n} Y_{i}$. Write also, $H_{n}(x)=\operatorname{Pr}\left(M_{n} \leqq B_{n} x\right)$ and for $n_{j}=\left[j k^{-1} n\right], j=1, \cdots, k,([u]$ here refers to the integer part of $u) H_{n, k}(x)=\operatorname{Pr}\left[\max \left(S_{n_{1}}, S_{n_{2}}, \cdots, S_{n_{k}}\right) \leqq B_{n} x\right]$. Then, since $B_{n}$ is of the form $n^{1 / \alpha} L(n)$ where $L(n)$ is slowly varying as $n \rightarrow \infty$ (Ibragimov and Linnik [10], Theorem 2.1.1), we have

$$
H_{n, k}(x)=\operatorname{Pr}\left(\operatorname{Max}\left(S_{n_{1}}, S_{n_{2}}, \cdots, S_{n_{k}}\right) \leqq n_{1}^{1 / \alpha} L\left(n_{1}\right)\left(\frac{n}{n_{1}}\right)^{1 / \alpha} \frac{L(n)}{L\left(n_{1}\right)} x\right) .
$$

Consequently, it follows from the multidimensional central limit theorem and Equation (2) that for $x>0$,

$$
\lim _{k \rightarrow \infty} \lim _{n \rightarrow \infty} H_{n, k}(x)=\lim _{k \rightarrow \infty} \operatorname{Pr}\left(\max _{1 \leqq j \leqq k} W_{j} \leqq k^{1 / \alpha} x\right)=H(x) .
$$

Now, for arbitrary $\varepsilon>0$, define

$$
X_{k n}= \begin{cases}X_{k} & \text { if }-K \varepsilon B_{n} \leqq X_{k} \leqq \varepsilon B_{n} \\ 0 & \text { otherwise }\end{cases}
$$

where $K=K(\varepsilon, n)$ is chosen such that $E X_{k n}=0$. It is easily seen that $K(\varepsilon, n) \rightarrow K_{0}$, a positive constant, as $n \rightarrow \infty$. Write $S_{n, m}=\sum_{k=1}^{m} X_{k n}$ where $m=n_{i+1}-r$ and $n_{i}<r \leqq n_{i+1}$. Then, if $N=n_{i+1}-n_{i}$, we have

$$
\begin{aligned}
\operatorname{Pr}(\mid & \left.S_{n_{i+1}}-S_{r} \mid>\varepsilon B_{n}\right)=\operatorname{Pr}\left(\left|S_{m}\right|>\varepsilon B_{n}\right) \\
& \leqq m\left[\operatorname{Pr}\left(X<-K \varepsilon B_{n}\right)+\operatorname{Pr}\left(X>\varepsilon B_{n}\right)\right]+\operatorname{Pr}\left(\left|S_{n, m}\right|>\varepsilon B_{n}\right) \\
& \leqq N\left[\operatorname{Pr}\left(X<-K \varepsilon B_{n}\right)+\operatorname{Pr}\left(X>\varepsilon B_{n}\right)\right]+\varepsilon^{-2} B_{n}^{-2} E S_{n, m}^{2} \\
& \leqq N\left[\operatorname{Pr}\left(X<-K \varepsilon B_{n}\right)+\operatorname{Pr}\left(X>\varepsilon B_{n}\right)\right]+N \varepsilon^{-2} B_{n}^{-2} E X_{k n}^{2},
\end{aligned}
$$


where we have used Chebyshev's inequality in obtaining the second last inequality. Now, $n B_{n}^{-2} E X_{k n}^{2} \leqq c_{1} \varepsilon^{2-\alpha}, n \operatorname{Pr}\left(X<-K \varepsilon B_{n}\right) \leqq c_{2} \varepsilon^{-\alpha}$, and $n \operatorname{Pr}\left(X>\varepsilon B_{n}\right) \leqq c_{3} \varepsilon^{-\alpha}$ as $n \rightarrow \infty$, where $c_{1}, c_{2}, c_{3}$ are positive constants (not depending on $\varepsilon$ ) (Feller [7], pages 304, 544-546) and, furthermore, $N \sim k^{-1} n$. It follows then, from (23), that we can choose a positive constant $c$ such that for all $i, r, n$,

$$
\operatorname{Pr}\left(\left|S_{n_{i}+1}-S_{r}\right|>\varepsilon B_{n}\right) \leqq c k^{-1} \varepsilon^{-\alpha} \text {. }
$$

Consequently for $x>0$,

$$
\begin{aligned}
\operatorname{Pr}\left(M_{n}>\right. & \left.B_{n} x\right)=\sum_{r=1}^{n} \operatorname{Pr}\left(\max _{1 \leqq j \leqq r-1} S_{j} \leqq B_{n} x, S_{r}>B_{n} x\right) \\
& =\sum_{i=0}^{k-1} \sum_{n_{i}<r \leqq n_{i+1}} \operatorname{Pr}\left(\max _{1 \leqq j \leqq r-1} S_{j} \leqq B_{n} x, S_{r}>B_{n} x,\left|S_{n_{i+1}}-S_{r}\right|>\varepsilon B_{n}\right) \\
& +\sum_{i=0}^{k-1} \sum_{n_{i}<r \leqq n_{i+1}} \operatorname{Pr}\left(\max _{1 \leqq j \leqq r-1} S_{j} \leqq B_{n} x, S_{r}>B_{n} x,\left|S_{n_{i+1}}-S_{r}\right| \leqq \varepsilon B_{n}\right) \\
& \leqq c k^{-1} \varepsilon^{-\alpha} \sum_{r=1}^{n} \operatorname{Pr}\left(\max _{1 \leqq j \leqq r-1} S_{j} \leqq B_{n} x, S_{r}>B_{n} x\right) \\
& +\sum_{i=0}^{k-1} \sum_{n_{i}<r \leqq n_{i}+1} \operatorname{Pr}\left(\max _{1 \leqq j \leqq r-1} S_{j} \leqq B_{n} x, S_{r}>B_{n} x,\left|S_{n_{i+1}}-S_{r}\right| \leqq \varepsilon B_{n}\right) \\
& \leqq c k^{-1} \varepsilon^{-\alpha}+1-H_{n, k}(x-\varepsilon),
\end{aligned}
$$

using (24). Finally, since $H_{n}(x) \leqq H_{n, k}(x)$, and with the aid of (25), we deduce that

$$
H_{n, k}(x-\varepsilon)-c k^{-1} \varepsilon^{-\alpha} \leqq H_{n}(x) \leqq H_{n, k}(x) .
$$

In view of (22), the result of the theorem is then immediate if we let $n \rightarrow \infty$ and then $k \rightarrow \infty$ in (26) since $H(x)$ is an absolutely continuous distribution function.

\section{Addendum}

The author has been informed by a referee that Theorems 3 and 4 of this paper are derivable from results of A. V. Skorokhod in "Random Processes with Independent Increments" Izdat. Nauk (Moscow) 1964 (in Russian). It has not been possible to compare methods due to the unobtainability of this book. A number of improvements to the exposition of this paper have been suggested by the referees and these the author gratefully acknow:ledges.

\section{References}

[1] BAXTER, G. AND DONSKER, M. D. (1957) On the distribution of the supremum functional for processes with stationary independent increments. Trans. Amer. Math. Soc. 85, 73-87.

[2] Chung-Ten, C. (1953) Explicit formula for the stable law of distribution. Acta Math. Sinica 3, 177-185. 
[3] Cramér, H. (1946) Mathematical Methods in Statistics. Princeton U. Press, Princeton.

[4] Darling, D. A. (1956) The maximum of sums of stable random variables. Trans. Amer. Math. Soc. 83, 164-169.

[51 ERDös, P. AND KAC, M. (1946) On certain limit theorems in the theory of probability. Bull. Amer. Math. Soc. 52, 292-302. 98-119.

[6] Feller, W. (1949) Fluctuation theory of recurrent events. Trans. Amer. Math. Soc. 67,

[7] Feller, W. (1966) An Introduction to Probability Theory and its Applications. Volume 2. Wiley, New York.

[8] Gradshteyn, I. S. And Ryzhik, I. M. (1965) Tables of Integrals, Series and Products. 4th Edition. Academic Press, New York.

[9] Heyde, C. C. (1967) A limit theorem for random walks with drift. J. Appl. Prob. 4, 144-150.

[10] Ibragimov, I. A. AND LinNik, Yu. V. (1965) Independent and Stationary Related Random Variables. Izd-vo "Nauka", Moscow. (In Russian). Paris.

[11] LÉvy, P. (1948) Processus Stochastiques et Mouvement Brownien. Gauthier Villars,

[12] Lukacs, E. (1960) Characteristic Functions. Griffin, London.

[13] Prabhu, N. U. (1965) Stochastic Processes. Macmillan, New York.

[14] RiCHTER, W. (1965) Limit theorems for sequences of random variables with sequences of random indices. Theor. Probability Appl. 10, 74-84. (English translation).

[15] Rogozin, B. A. (1964) On the distribution of the first jump. Theor. Probability Appl. 9, 450-465. (English translation).

[16] Spitzer, F. (1964) Principles of Random Walk. Van Nostrand, New York.

[17] Widder, D. V. (1941) The Laplace Transform. Princeton U. Press, Princeton.

[18] Zolotarev, V. M. (1957) Mellin-Stieltjes transforms in probability theory. Theor. Probability Appl. 2, 433-460. (English translation). 